\title{
La Paleodieta de Cinco Especies de Mamíferos Herbívoros Rancholabreanos de Valsequillo (Puebla, México)
}

\author{
The Paleodietof Five Species of Rancholabrean Mammals Herbivores from Valsequillo \\ (Puebla, México) \\ Víctor A. Pérez-Crespo ${ }^{\mathrm{i}}$, Joaquín Arroyo-Cabrales ${ }^{\mathrm{ii}}$, Luis M. Alva-Valdivia ${ }^{\mathrm{iii}}$, Pedro \\ Morales-Puente, Edith Cienfuegos-Alvarado, Francisco J. Otero ${ }^{\text {iv }}$ y Patricia Ochoa- \\ Castillov $^{v}$
}

\section{RESUMEN}

Usando las relaciones isotópicas de ${ }^{13} \mathrm{C} /{ }^{12} \mathrm{C} y{ }^{18} \mathrm{O} /{ }^{16} \mathrm{O}$ presentes en el esmalte y la osteodentina, se infirió la dieta y el ambiente en el cual vivieron cinco especies extintas de herbívoros procedentes del Pleistoceno Tardío deValsequillo, Puebla, México. Los caballos, los mamutes y el perezoso mostraban una dieta basada exclusivamente en plantas $\mathrm{C}_{4}$, mientras que el carpincho y los gonfoterios exhibían una dieta mixta $\mathrm{C}_{3} / \mathrm{C}_{4}$. El tipo de vegetación en el cual estos animales habitaron fue abierto con poca cobertura arbórea, misma que ha sido propuesta en otros estudios usando la presencia de algunas especies de micromamíferos encontradosen la zona.

Palabras Clave:Valsequillo, Dieta, Hábitat, Mamíferos, Isótopos Estables

\section{ABSTRACT}

Through the use of the ${ }^{13} \mathrm{C} /{ }^{12} \mathrm{C}$ and ${ }^{18} \mathrm{O} /{ }^{16} \mathrm{O}$ isotopic relationships found at enamel and osteodentine, here it is inferred the diet and preferred habitat in which five extinct herbivore species from the Late Pleistocene of Valsequillo, Puebla, México. The horses, mammoths, and sloths showed a diet mainly based in $\mathrm{C}_{4}$, meanwhile the capybaraand gomphotheres were mixed $\mathrm{C}_{3} / \mathrm{C}_{4}$

i Posgrado en Ciencias Biológicas, UNAM. Ciudad Universitaria, Del. Coyoacán, 04510, México, D. F. Correo-e: vapc79@gmail.com.

ii Laboratorio de Arqueozoología "M. en C. Ticul Álvarez Solórzano", Subdirección de Laboratorios y Apoyo Académico, INAH. Moneda 16 Col. Centro, 06060, México, D. F. Correoe: arromatu@hotmail.com

iii Laboratorio de Paleomagnetismo, Instituto de Geofísica, UNAM. Ciudad Universitaria, Del. Coyoacán, 04510, México, D. F. Correo-e: lalva@tonatiuh.igeofcu.unam.mx

iv Instituto de Geología, Universidad Nacional Autónoma de México, Circuito de la Investigación Científica S/N, Ciudad Universitaria, Del. Coyoacán, 04510 México, D. F. Correo-e: pedro_morales1@me.com, edithca@geol-sun.igeolcu.unam. $\mathrm{mx}$, fotero@geologia.unam.mx

v Subdirección de Arqueología, Museo Nacional de Antropología, INAH, Av. Paseo de la Reforma y Calzada Gandhi, Col. Chapultepec Polanco, 11560 México, D. F. Correo-e: patricia_ochoa_cast@yahoo.com

Recibido: 25-11-2012 Revisado: 05-06-2013 Aceptado:02-02-201 
feeders. The vegetation in which those animals lived was open with some trees, similar to the one proposed in other studies based in some macromammals species found in the locality.

Key Words:Valsequillo, Diet, Habitat, Mammals, Stable Isotopes

\section{INTRODUCCIÓN}

Valsequillo, Puebla, es una localidad donde se han encontrado posibles rastros de actividad humana asociada a restos de mamíferos del Pleistoceno Tardío. Por tal motivo, se han realizado en la zona diversos trabajos arqueológicos (OchoaCastillo et al., 2003;),bioestratigráficos(Pichar do 2000),paleoclimáticos (Stevens et al. 2012),y paleontológicos.

Para este ultimo tópico, la mayor parte de los trabajos se han enfocado a describir los taxa que se han encontrado en la zona (González et al. 2006) y,únicamente Cruz-Muñoz et al. (2009), usando los restos de los micromamíferos hallados en la región, propusieron un posible ambiente del sitio durante el Pleistoceno Tardío. Sin embargo, dado que en el sitio también existieron mamíferos herbív oros de tallas medianas y grande, en este trabajo se determina el tipo de dieta y el hábitat de estas especies usando para ello las proporciones isotópicas ${ }^{13} \mathrm{C} /{ }^{12} \mathrm{C}$ y ${ }^{18} \mathrm{O} /{ }^{16} \mathrm{O}$ presentes en el apatito del esmalte $\mathrm{o}$ la osteodentina.

\section{Marcadores biogeoquímicos $\delta^{13} \mathrm{C}$ y $\delta^{18} \mathrm{O}$}

Existen tres vías fotosintéticas en las plantas, que pueden ser reconocidas por los valores de $\delta^{13} \mathrm{C}$ : las plantas $\mathrm{C}_{3}(-22 \%$ o a $-30 \%$ ), principalmente árboles y arbustos dicotiledóneos y algunos pastos de zonas frías; las $\mathrm{C}_{4}(-10 \%$ a $-14 \%$ ), pastos monocotiledóneos, pteridófitas y algunos árboles y arbustos dicotiledóneos de zonas cálidas; y la vía CAM presente en bromelias, cactáceas, orquídeas y otras plantas suculentas, muestra valores de $-10 \%$ a $-30 \%$ o por lo que no se puede distinguir de las otras vías fotosintéticas (Smith y Epstein 197I,Keeley y Rundel 2003).

Los valores anteriores se ven reflejados en los animales herbívoros con un incremento (enriquecimiento) del $14 \%$ o con respecto de los valores de la planta consumida (Sánchez 2005), quedando para las especies ramoneadoras valores de -9 a $-19 \%$; los pacedores con -2 a $+2 \%$ y, en los organismos con dieta mixta los valores son de -9 a $-2 \%$ (MacFadden y Cerling, 1996).
En el caso del oxígeno,este ingresa principalmente a través del agua ingerida y,en menor medida por medio del agua que se obtiene de los alimentos, así como el que se inhala, hallándose en balancecon el oxígeno que se pierdecomo agua por mediodel sudor, las heces, la orina y la exhalación (Sánchez, 2005); factores como la fisiología, la dieta y el tipo de hábitat de cada especie, así como el clima intervienen en este balance, aunque en la caso de los mamíferos de talla grande, depende principalmente de este último factor (Bryantet al. 1996; Koch, 1998). Dado que el agua ingerida es la principal fuente de oxígeno, la $\delta^{18} \mathrm{O}$ se halla influenciada por factores como la altitud, la latitud, la distancia que existe entre el mar hacia tierra firme, pero principalmente por la temperatura media anual, por lo que la relación isotópica del oxígeno es usada para inferir las condiciones climáticas en el pasado y su uso, en conjunto con las relaciones isotópicas de carbono, permite deducir el hábitat que una especie prefería(Schoeningeret al. 2000; MacFaddenet al. $2004)$.

\section{MATERIALESY MÉTODOS}

\section{Lugar de estudio}

La localidad de Valsequillo se localiza entre los $18^{\circ} 54^{\prime} \mathrm{N}$ y $98^{\circ} 10^{\prime} \mathrm{O}$ a $2040 \mathrm{msnm}$ y $15 \mathrm{~km}$ al sur de la ciudad de Puebla, México (Cruz-Muñozet al. 2009) (Figura I). En la zona se han encontrado rastros de actividad humana y restos fósiles de diversos mamíferos herbívoros y carnívoros entre los que destacan carpinchos (Neochoerussp.), caballos (Equussp.), gonfoterios (Stegomastodonsp.), mamutes (Mammuthuscolumbi) y perezosos terrestres (Paramylodonharlani), así como diversas especies de lagomorfos y roedores, en cuatro diferentes sitios llamados El Horno, El Mirador, Hueyatlaco y Tecacaxcoy la localidad ha sido fechada en 20,780 A.P; sin embargo, es importante indicar que se desconoce la procedencia exacta de la mayoría de los restos y, si estos fueron contemporáneos o representan diversas temporalidades(González et al. 2006). 


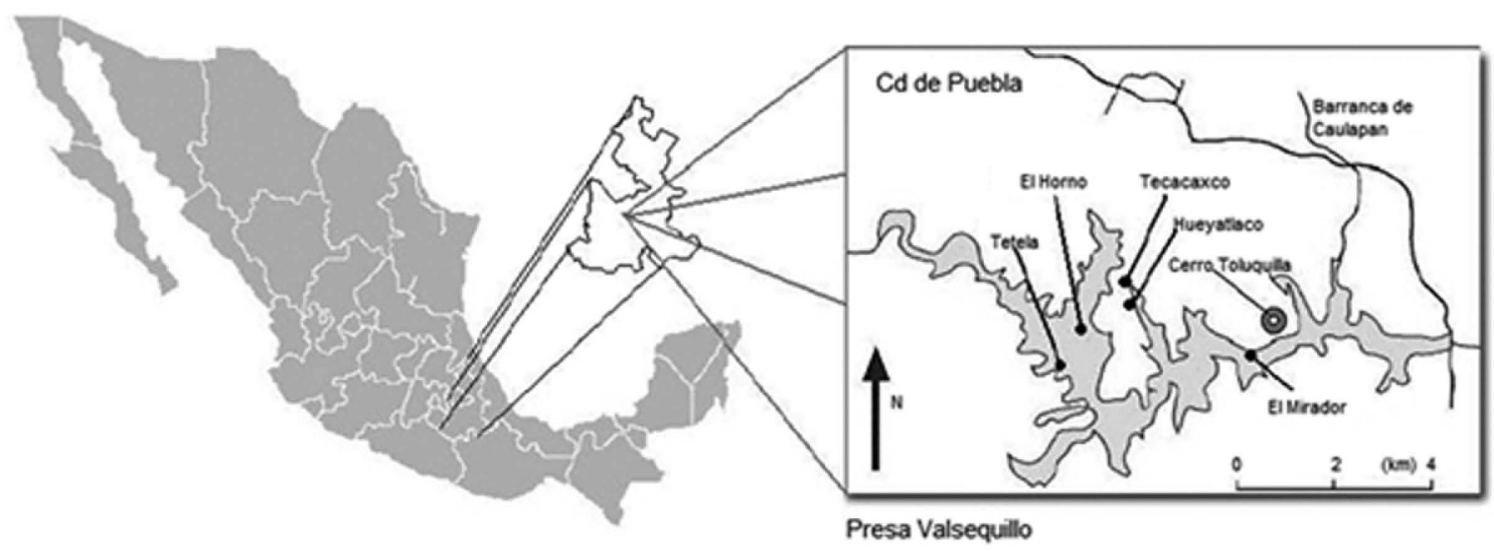

Figura 1: Mapa de Valsequillo con los cuatro sitios donde se han colectado material fósil y arqueológico. Tomado de Cruz-Muñoz et al. (2009).

Figure 1: Valsequillo map with four sites where has found collected fossil and archeological material. Take of Cruz-Muñoz et al. (2009).

\section{Extracción y preparación de muestras}

Se tomó una muestra de esmalte dental y de osteodentina de un carpincho, dos caballos, seis gonfoterios, dos mamutes y un perezosoterrestre Paramylodonharlani (tablal), que se hallan depositados en la colección Paleontológica del Laboratorio de Arqueozoología "M. en C. Ticul Álvarez Solórzano", Subdirección de Laboratorios y Apoyo Académico del Instituto Nacional de Antropología e Historia, pero carecen de número de catalogo, así como datos que indiquen de cual de los cuatro sitios que existen en Valsequillo proceden.

Una vez obtenidas las muestras, éstas fueron preparadas siguiendo el método de Koch et al. (1997) y enviadas al Laboratorio de Isótopos Estables del Instituto de Geología de la UNAM donde fueron analizadas de acuerdo al procedimiento descrito por Révész y Landwehr (2002) y normalizados de acuerdo a Coplen (1988), Coplenet al. (2006) y Werner y Brand (200I).

\section{Análisis estadístico}

Se obtuvo la media y la desviación estándar de los valores de $\delta^{13} \mathrm{C}$ y $\delta^{18} \mathrm{O}$ de cada una de las especies analizadas; en el caso de los valores de $\delta^{13} \mathrm{C}$ los mismos fueron contrastados con los propuestospor MacFadden y Cerling (1996) para determinar el tipo de dieta de cada una de las especies. Posteriormente, estos valores fueron comparados por medio de un Análisis de Varianza (ANOVA) y la prueba de TukeyKramer para detectar diferencias o semejanzas entre ellos (Hammer y Harper 2006).

Finalmente, los valores isotópicos de carbono fueron graficados en conjunto con la $\delta^{18} \mathrm{O}$ para inferir el tipo de hábitat que los taxa bajo estudio preferían, siguiendo el modelo propuesto por White et al. (2009). Secord et al. (2008) han indicado que los valores $\delta^{13} \mathrm{C}$ más negativos, $-19 \%$, se encuentran en zonas con una importante cobertura arbórea, pero al disminuir esta, los valores isotópicos de carbono se van haciendo más positivos. Quade et al. (1995) señalan que las plantas que viven en zonas de vegetación abiertas experimentan una evapotranspiración más alta que las que viven en zonas cerradas, por lo que sus $\delta^{18} \mathrm{O}$ son más positivos que las otras. Sin embargo, dado que la relación isotópica del oxígeno está afectada por diversos factores, esta relación no se cumple en muchas ocasiones.Adicionalmente debe considerarse que este modelo solo permite indicar si el tipo de vegetación fue cerrado o abierto, por lo que para determinar con mayor detalle el tipo de vegetación que existió en la zona, se usó la información de otros trabajos que se han realizado en la zona.El nivel de significancia empleado fue de $p<0.05$ y el software empleado fue NCCS and PASS (Hintze 2004). 


\section{RESULTADOS}

Se puede observar que en el caso de los caballos su promedio de $\delta^{13} \mathrm{C}$ es de $-0.98 \%$, mientras que para la $\delta^{18} \mathrm{O}$ la media es de $-7.04 \%$. Para los gonfoterios los valores promedios de $\delta^{13} \mathrm{C}$ y $\delta^{18} \mathrm{O}$ son de $-4.25 \%$ \% $-7.75 \%$; en cambio las medias de $\delta^{13} \mathrm{C}$ y $\delta^{18} \mathrm{O}$ de los mamutesson de $-1.82 \%$ y $-6.11 \%$ respectivamente. El carpincho muestra valores isotópicos de carbono de $-2.11 \%$ y de oxígeno de $-6.5 \%$, mientras que el perezoso terrestre su $\delta^{13} \mathrm{C}$ es de $-4.7 \%$ y de $\delta^{18} \mathrm{O}-6.5 \%$ (Tablas I y2)

\begin{tabular}{|l|c|c|}
\hline & $\delta^{13} \mathrm{C}$ & $\delta^{18} \mathrm{O}$ \\
\hline Equussp. & -0.55 & -7.4 \\
\hline Equussp. & -1.42 & -6.69 \\
\hline Mammuthuscolumbi & -0.99 & -4.7 \\
\hline Mammuthuscolumbi & -2.65 & -7.53 \\
\hline Neochoerussp. & -2.11 & -6.5 \\
\hline Paramylodonharlani & -1.8 & -4.7 \\
\hline Stegomastodonsp. & -3.28 & -4.79 \\
\hline Stegomastodonsp. & -4.08 & -8.68 \\
\hline Stegomastodonsp. & -4.39 & -9.53 \\
\hline Stegomastodonsp. & -4.46 & -7.65 \\
\hline Stegomastodonsp. & -4.8 & -8.09 \\
\hline Stegomastodonsp. & -4.54 & -7.77 \\
\hline
\end{tabular}

Tabla 1: Valores isotópicos de los ejemplares de Valsequillo. Table 1: Isotopic values of Valsequillo mammals.

\begin{tabular}{|l|l|l|l|l|}
\hline & $\mathrm{n}$ & Promedio & Mínimo & Máximo \\
\hline Equussp. & 2 & $\begin{array}{l}\delta^{13} \mathrm{C}:-0.98 \\
\delta^{18} \mathrm{O}:-7.04\end{array}$ & $\begin{array}{l}\delta^{13} \mathrm{C}:-1.42 \\
\delta^{18} \mathrm{O}:-7.4\end{array}$ & $\begin{array}{l}\delta^{13} \mathrm{C}:-0.55 \\
\delta^{18} \mathrm{O}:-6.69\end{array}$ \\
\hline Mammuthuscolumbi & 2 & $\begin{array}{l}\delta^{13} \mathrm{C}:-1.82 \\
\delta^{18} \mathrm{O}:-6.11\end{array}$ & $\begin{array}{l}\delta^{13} \mathrm{C}:-2.65 \\
\delta^{88} \mathrm{O}:-7.53\end{array}$ & $\begin{array}{l}\delta^{13} \mathrm{C}:-0.99 \\
\delta^{18} \mathrm{O}:-4.7\end{array}$ \\
\hline Neochoerussp. & $\mathrm{I}$ & $\begin{array}{l}\delta^{13} \mathrm{C}:-2.1 \mathrm{l} \\
\delta^{18} \mathrm{O}:-6.5\end{array}$ & & \\
\hline Paramylodonharlani & $\mathrm{I}$ & $\begin{array}{l}\delta^{13} \mathrm{C}:-1.8 \\
\delta^{18} \mathrm{O}:-4.7\end{array}$ & & \\
\hline Stegomastodonsp. & 6 & $\begin{array}{l}\delta^{13} \mathrm{C}:-4.25 \\
\delta^{18} \mathrm{O}:-7.75\end{array}$ & $\begin{array}{l}\delta^{13} \mathrm{C}:-4.8 \\
\delta^{18} \mathrm{O}:-9.53\end{array}$ & $\begin{array}{l}\delta^{13} \mathrm{C}:-3.28 \\
\delta^{18} \mathrm{O}:-4.79\end{array}$ \\
\hline
\end{tabular}

Tabla 2: Valores promedio y valores máximos y mínimos de $\delta^{13} \mathrm{C}$ y $\delta^{18} \mathrm{O}$ de los herbívoros de Valsequillo: $\mathrm{n}$ : número de individuos.

Table 2: Average values and maximun and minimun of herbivores

$\delta^{13} C y \delta^{18} O$ values from Valsequillo.n: individual number.

El ANOVA indica que existen diferencias entre los valores de $\delta^{13} \mathrm{C}$ (g.l: II, F: 12.43 y $\mathrm{p}<0.002684^{*}$ ) y la prueba de Tukey-Kramer señala que los gonfoterios son estadísticamente diferentes a los caballos y los mamutes. El gráfico realizado con los valores de $\delta^{13} \mathrm{C}$ y $\delta^{18} \mathrm{O}$ muestra que la mayor parte de los gonfoterios forman un grupo, mientras el resto de las especies y un gonfoterio se distribuyen en la mitad del gráfico (Figura 2).

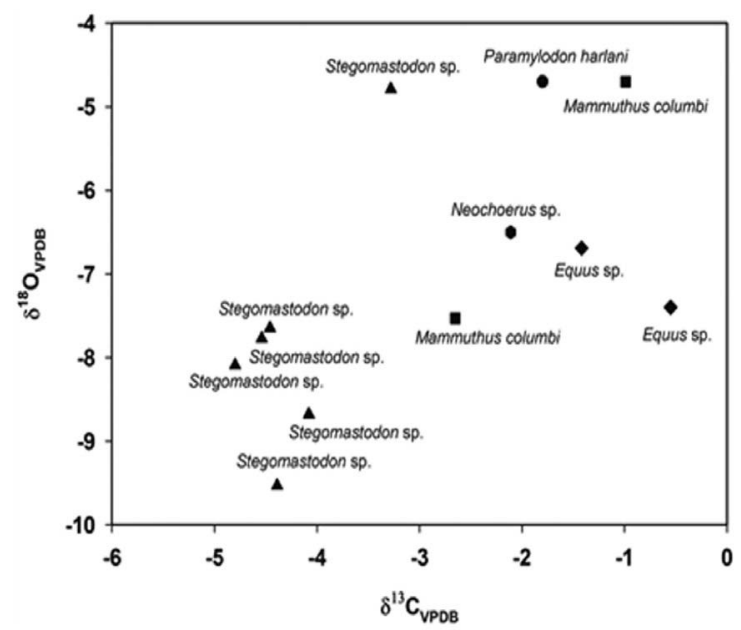

Figura 2: Gráfico de los valores de $\delta^{13} \mathrm{C}$ y $\delta^{18} \mathrm{O}$ de los ejemplares de Valsequillo.

Figure 2: Graphic of species $\delta^{13} \mathrm{C}$ and $\delta^{18} \mathrm{O}$ values of Valsequillo

\section{DISCUSIÓN}

\section{Hábito alimentario}

Los caballos muestreados fueron individuos con una dieta exclusivamente basada en plantas $\mathrm{C}_{4}$ los trabajos realizados por Pérez-Crespo et al. (2009, $2012 a)$ con équidos provenientes de Cedral y la Laguna de las Cruces, San Luis Potosí, México, muestran que estaspoblaciones exhibían en promedio una dieta mixta $\mathrm{C}_{3} / \mathrm{C}_{4}$, pero existiendo algunos individuos con una dieta exclusivamente $\mathrm{C}_{4}$; en cambio Nunez et al. (2010) encuentran en los caballos de Terapa, Sonora, una dieta exclusivamente pacedora. Bravo-Cuevas et al. (20II) han indicado que los équidos mexicanos no se alimentaban exclusivamente de pastos, sino que eran más flexibles en su dieta, variando la proporción de pastos, hojas de árboles y arbustos, dependiendo de la disponibilidad de éstos en la zona que habitaran.

Para el carpincho, su valor isotópico de $\delta^{13} \mathrm{C}$ indica una dieta mixta $\mathrm{C}_{3 /} \mathrm{C}_{4}$, pero con un importante consumo de plantas $\mathrm{C}_{4}$; similar a lo encontrado en un ejemplar de Bolivia (MacFadden y Shockey 1997) y las observaciones realizadas en los capibaras modernos han demostrado que esta especie se alimenta principalmente de poaceas (Quintana et al. 1998). 
En el caso de los mamutes, el tipo de dieta en promedio es del tipo pacedor, con un individuo con una dieta mixta $\mathrm{C}_{3} / \mathrm{C}_{4}$ y otro completamente basada en plantas $C_{4}$. Pérez-Crespo et al. (20l2b) y Rivalset al. (2012) quienes analizan la dieta y el hábitat de esta especie en México y EUA, respectivamente,encuentran que este organismo exhibía en promedio una dieta mixta $\mathrm{C}_{3} / \mathrm{C}_{4}$, pero había individuos que se alimentaban exclusivamente de plantas $\mathrm{C}_{4}$. En el caso de los ejemplares de Valsequillo, estos siguen la misma tendencia alimentaria, que el resto de los mamutes mexicanos

La dieta del perezoso terrestre es del tipo pacedor; los trabajos morfológicos realizados por Bargoet al. (2006) han sugerido que esta especie se alimentaba de pastos y otras herbáceas, como lo sugiere el ejemplar de Valsequillo. Sin embargo, McDonald y Pelikan (2006) han señalado que esta especie se alimentaba además de herbáceas, de hojas de árboles y arbustos, algo que es observado en los valores de $\delta^{13} \mathrm{C}$ de los individuos de esta especie muestreados en Terapa, Sonora (Nunezet al. 2010). Esta variación en la dieta del ejemplar de Valsequillo con respecto a los de Sonora, pudiera deberse a que en Valsequillo existieran más plantas $C_{4}$ que en Terapa durante el Rancholabreano, lo cual también explicaría que los caballos del sitio mostraran una dieta completamente pacedora.

En contraste, la $\delta^{13} \mathrm{C}$ promedio de los gonfoterios indica una dieta mixta $\mathrm{C}_{3} / \mathrm{C}_{4}$; Kurtén $\mathrm{y}$ Anderson (1980) propusieron que los gonfoterios americanos eran ramoneadores, lo cual se basa en los molares braquiodontos que estos animales poseen. Sin embargo, Arroyo-Cabrales et al. (2010), con base a los trabajos isotópicos realizados por Sánchez et al. (2004) con las especies de gonfoterios sudamericanas, han sugerido que el género Stegomastodonen México era un organismo de dieta mixta. Los resultados de los ejemplares deValsequillo apoyan esta propuesta.

\section{Hábitat}

El gráfico entre los valores de $\delta^{13} \mathrm{C}$ y $\delta^{18} \mathrm{O}$ de las especies analizadas indica que en Valsequillo, se desarrolló una zona abierta pero con algunosárboles, esto último basado en la información isotópica de la mayoría de los Stegomastodon, los cuales forman un grupo separado de las otras especies. Dado que en Valsequillo existían otras especies que habitaban en zonas abiertas como los berrendos, los bisontes, los camellos, los gliptodontes y los pampaterios, junto con algunas especies de zonas cerradas como son los jabalíes, las llamas y el perezoso Megalonyxjeffersoni(Jonhnsonet al. 2006), se puede proponer que lo encontrado por los datos isotópicos es correcto.

Cruz-Muñoz et al. (2009), basándose en la presencia de diversas especies de micromamíferos como Cynomysmexicanusy Romerolagusdiazi, han sugerido la existencia de un matorral xerófilo con un bosque de coníferas;Stevens et al. (20I2), quienes reconstruyen el clima del sitio desde los 35 000 A.P. hasta los 20000 A. P. indican que hubo un incremento en la aridez en la zona y posteriormente el clima se hizo más húmedo, aunque finalmente el registro se trunca. Estas fluctuaciones en el clima provocaron que tanto el matorral xerófilo como el bosque de coníferas se expandieran y contrajeran como respuesta a estos cambios el clima. Dado que los matorrales xerófilos son un tipo de vegetación abierta aunque dominada por plantas CAM (Rzedowski, 1981), los datos obtenidos por los isótopos estables, concuerdan con lo propuesto por Cruz-Muñoz et al. (2009); sin embargo, al igual que este trabajo, esto debe ser corroborado con estudios bioestratigráficos que indiquen si todos los restos de las especies encontradas son contemporáneos o pertenecieron a periodos distintos.

\section{CONCLUSIONES}

Los herbívoros de Valsequillo se dividen en dos categorías alimentarias: organismos pacedores, representados por los caballos, los mamutes y el perezoso y animales con una dieta mixta $C_{3} / C_{4}$, como el carpincho y los gonfoterios. La zona durante el rancholabreanodebió haber sido una zona abierta con algunosárboles, confirmado esto por la presencia de otras especies de herbívoros y los restos de micromamíferos hallados en Valsequillo. Sin embargo, es importante contar con el control estratigráfico que permita indicar si las especies halladas en la zona son contemporáneas o no $y$, de esta manera tener un panorama más amplio de las condiciones en las 
cuales vivieron los primeros habitantes de México durante este periodo, en esta zona.

\section{Agradecimientos: Al Consejo Nacional} de Arqueología del INAH por el permiso para tomar las muestras de esmalte dental. Al Consejo Nacional de Ciencia y Tecnología y al Posgrado en Ciencias Biológicas UNAM por la beca de estudios otorgada al primer autor (\# 20044I) y por el apoyo otorgado (\#132620). Al Laboratorio de Isótopos Estables del Instituto de Geología de la UNAM y R. Puente M. por analizar las muestras. A dos anónimos revisores cuyos comentarios enriquecieron el presente trabajo.

\section{BIBLIOGRAFÍA}

Arroyo-Cabrales, J., O. J. Polaco, E.Johnsonyl. Ferrusquía-Villafranca.2010. "A perspective on mammal biodiversity and zoogeography in the Late Pleistocene of México". Quaternary International 212: 187-197.

Bargo, S. M., G. De luliis y S. F. Vizcaíno.2006. "Hypsodonty in Pleistocene ground sloths". Acta PaleontologicaPolonica 51:53-61.

Bravo-Cuevas, V. M., E. Jimenez-Hidalgo y J. Priego-Vargas. 2011. "Taxonomía y hábitos alimentarios de Equusconversidens (Perissodactyla, Equidae) del Pleistoceno Tardío (Rancholabreano) de Hidalgo, centro de México". Revista Mexicana de Ciencias Geológicas 28:65-82.

Bryant, J. D., P. N.Froelich, W. J.Showers y B. J.Genna.1996. "Biologic and climate signals in the oxygen isotopic composition of Eocene-Oligocene equid enamel phosphate". Paleogeography, Palaeoclimatology, Palaeoecology 126:75-89.

Coplen, T. 1988. "Normalization of oxygen and hydrogen isotope data". Chemical Geology (Isotope Geoscience Section) 72: 293297.

Coplen, T. W. A. Brand, M. Gehre, M. Gröning, A. J. Meijer Harro, B. Toman y R. M. Verkouteren. 2006. "New Guidelines for $\delta^{13} \mathrm{C}$ measurements". AnalyticalChemistry 78: 2439-244I.

Cruz-Muñoz, V. J. Arroyo-Cabrales y R. W. Graham.2009. "Rodents and lagomorphs (Mammalia) from the Late-Pleistocene deposits at Valsequillo, Puebla, México". Current Research in the Pleistocene26:147-149.

González, S., D. Huddart y M. R. Bennett.2006. "Valsequillo Pleistocene archeology and dating: ongoing controversy in Central Mexico". World Archeology 38:6 I I -627.

Hammer, ø. y D. Harper. 2006. Paleontological Data Analysis. Blackwell Publishing.

Hintze, J. 2004. NCSS and PASS.Number Cruncher Stastical System. Kaysville, UTHA.WWW.NCSS.COM.

Johnson, E., J. Arroyo-Cabrales y O. J. Polaco.2006. "Climate, environment, and game animal resources of the Late Pleistocene Mexican grassland”. En El hombre temprano en América y sus implicaciones en el poblamiento de la cuenca de México, editado porL. J. C. Jiménez, S. González, J.A. Pompa y Padilla y P. F. Ortiz, Pp. 23I-245. .INAH, México.

Keeley, J. E. y P. W. Rundel.2003. "Evolution of CAM and C4 carbon-concentrating mechanisms". International Journal Plants of Sciencel 64 (supplement 3):S55-S77.

Koch, P. L. 1998. "Isotopic reconstruction of past continental environments".Annual Review Earth Planetary Science 26:573-6I3

Koch, P. L., N. Tuross y M. L. Fogel. 1997.'The effects of sample treatment and diagenesis on the isotopic integrity of carbon in biogenic hydroxylapatite".Journal of Archaeological Science 24:417-429.

Kurtén, B. y E. Anderson. 1980. Pleistocene Mammals of North America. Columbia University Press, USA.

MacFadden, B. J. y T. E. Cerling. 1996. "Mammalian herbivore communities, ancient feeding ecology, and carbon isotopes: a 10 million-year sequence from the Neogene of Florida". Journal of Vertebrate Paleontology 16:103-115.

MacFadden, B. J. y B. J.Shockey. 1997. "Feeding ecology and niche differentiation of Pleistocene mammalian herbivores from Tarija, Bolivia: morphological and isotopic evidence". Paleobiology 23:77-100.

MacFadden, B. J., P. Higgins, M. Clementz y D. S. Jones. 2004. "Diets, habitat preferences, and niche differentiation of Cenozoic sirenians from Florida: Evidence from stable isotopes". Paleobiology 30:297-324.

McDonald, H. G. y S. Pelikan. 2006."Mammoths and mylodonts: Exotic species from two different continents in North American Pleistocene faunas". Quaternary International 142-I43: 229-24I.

Nunez, E. F., B. J. MacFadden, J. I. Mead y A. Baez, A. 2010. "Ancient forests and grasslands in the desert: Diet and habitat of Late Pleistocene mammals from Norhcentral Sonora, México". Paleogeography, Palaeoclimatology, Palaeoecology297:391-400.

Ochoa-Castillo, P., M. Pérez-Campa, A. L. Martín del Pozzo y J. Arroyo-Cabrales.2003. "New excavations in Valsequillo, Puebla, México". CurrentResearch in thePleistocene 20:61-62.

Pérez-Crespo, v. A., B. Sánchez-Chillón, J. Arroyo-Cabrales, M. T. Alberdi, O.J. Polaco, A. Santos-Moreno, M.Benammi, P. Morales-Puente y E. Cienfuegos-Alvarado. 2009."La dieta y el hábitat del mamut y los caballos del Pleistoceno tardío de El Cedral con base en isótopos estables $\left(\delta^{13} \mathrm{C}, \delta^{18} \mathrm{O}\right)$ ". Revista Mexicana de Ciencias Geológicas 26:347-355.

Pérez-Crespo, V. A., J. Arroyo-Cabrales, L. M. Alva-Valdivia, P. Morales-Puente y E. CienfuegosAlvarado. 2012a. "Datos isotópicos $\left(\delta^{13} \mathrm{C}, \delta^{18} \mathrm{O}\right)$ de la fauna Pleistocénica de la Laguna de las Cruces, San Luis Potosí, México". Revista Mexicana de Ciencias Geológicas 29:299-307.

Pérez-Crespo, V. A., J. Arroyo-Cabrales, M. Benammi, E., O. J. Polaco, A. Santos-Moreno, P. Morales-Puente y E. Cienfuegos-Alvarado. 20I2b. "Geographic variation of diet and habitat of the Mexican populations of Columbian Mammoth (Mammuthuscolumbi)". Quaternary International 276-277:8-16. 
Pichardo, M. 2000. "Valsequillo biostratigraphy III: Equid ecospecies in paleoindian sites". AnthropologischerAnzeiger 58: 275-298.

Quade, J., T. E. Cerling, P. Andrews y B. Alpagut. 1995. "Paleodietary reconstruction of Miocene faunas from Pasalar, Turkey using stable carbon and oxygen isotopes of fossil tooth enamel". Journal of Human Evolution 28:373-384.

Quintana, R. D., S. Monge y A. I.Mavárez.1998. "Composición y diversidad de la dieta del capibara (Hydrochaerishydrochaeris) y del ganado doméstico en un agroecosistema de la región central de entre ríos, Argentina". Ecotrópicos I 1:33-44.

Révész, K. M. y J. M. Landwehr.2002. " $\delta^{13} C$ and $\delta^{18} \mathrm{O}$ isotopic composition of $\mathrm{CaCO}_{3}$ measured by continuous flow isotope ratio mass spectrometry: statiscal evaluation and verification by application to Devils Hole core $\mathrm{DH}-\mathrm{II}$ calcite". Rapid Communications in Mass Spectrometry 16:1012-2114.

Rivals, F., G. Semprebon y A. Lister.20I2. "An examination of dietary diversity patterns in Pleistocene proboscideans (Mammuthus, Paloeoloxodon, and Mammut) from Europe and North America as revealed by dental microwear". Quaternary International 255:188-195. México.

Rzedowski, J. 198I. Vegetación de México. Editorial Limusa,

Sánchez, B. 2005. "Reconstrucción del ambiente de mamíferos extintos a partir del análisis isotópico de los restos esqueléticos". En Nuevas técnicas metodológicas aplicadas al estudio de los sistemas ambientales: los isótopos estables, editado por P.Alcorno, R. Redondo y J. Toledo, pp. 49-64. Universidad Autónoma de Madrid, España.

Sánchez, B., J. L. Prado, J. L. y M. T. Alberdi.2004."'Feeding ecology, dispersal, and extinction of South American Pleistocene gomphotheres (Gomphotheriidae, Proboscidea)".Paleobiology 30:146-161.

Schoeninger, M. J., M. J. Kohn y J. W. Valley. 2000. "Tooth oxygen isotope ratios as paleoclimate monitors in arid ecosystems". En Biogeochemical approaches to paleodietary analysis, editadopor S. H. Ambrosey M. A. Katzenberg, Pp. I I7-I39. Kluwer Academic/Plenum Publisher, New York.

Secord, R., W. L. Scott y A. Chew. 2008. "Stable isotopes in early Eocene mammals as indicator of forest canopy structure and resource partitioning". Paleobiology 34: 282-300.

Smith, B. N. y S. Epstein. 1971. "Two categories of ${ }^{13} \mathrm{C} /{ }^{12} \mathrm{C}$ ratios for higher plants". Plant Physiology 47:380-384.

Stevens, R. E., S. E. Metcalfe, M. J. Leng, A. L Lamb, H. J. Sloane, E.Naranjo yS. Gonzalez.20I2. "Reconstruction of late Pleistocene climate in the Valsequillo Basin (Central México) through isotopic analysis of terrestrial and freshwater snails". Palaeogeography, Palaeoclimatology, Palaeoecology 319-320:16-27.

Werner, R. A. y W. A. Brand. 200I. "Referencing strategies and techniques in stable isotope ratio analysis". Rapid Communications in Mass Spectrometry I5: 501-5I9.

White, T. D., B. Asfaw, Y. Beyene, Y. Haile-Salassie, C. OwenLevejov, G. Suw y G. WoldeGabriel.2009."Ardipithecusramidus and thepaleobiology of Earlyhominds”. Science326:75-86.. 